01

\title{
Дисперсионные силы между металлической и диэлектрической пластинами, разделенными магнитной жидкостью
}

\author{
(C) Е.Н. Величко, ${ }^{1}$ Г.Л. Климчицкая,, ${ }^{1,2}$ В.М. Мостепаненко ${ }^{1,2}$ \\ ${ }^{1}$ Институт ффизики, нанотехнологий и телекоммуникаций, \\ Санкт-Петербургский политехнический университет Петра Великого, \\ 195251 Санкт-Петербург, Россия \\ 2 Главная (Пулковская) астрономическая обсерватория РАН, \\ 196140 Санкт-Петербург, Россия \\ e-mail: velichko-spbstu@yandex.ru,g_klimchitskaya@mail.ru,vmostepa@rambler.ru
}

Поступило в Редакцию 20 декабря 2018 г.

В окончательной редакции 20 декабря 2018 г.

Принято к публикации 24 марта 2019 г.

\begin{abstract}
Формализм теории Лифшица применен для определения давления дисперсионных сил между металлической и диэлектрической пластинами, разделенными тонким слоем магнитной жидкости. Численные расчеты проведены для пластин из золота, кварцевого стекла и магнитной жидкости, состоящей из керосина и наночастиц магнетита, при комнатной температуре. Для этого использованы известные представления диэлектрических свойств золота и кварцевого стекла вдоль мнимой оси частот, а также получены соответствующие представления для магнетита и керосина. Дисперсионное давление исследовано как функция расстояния между пластинами, объемной доли частиц магнетита в магнитной жидкости и их диаметра. На достаточно больших расстояниях между пластинами для него выведены простые аналитические формулы. Обсуждены возможные приложения полученных результатов.
\end{abstract}

Ключевые слова: дисперсионные силы, магнитные жидкости, теория Лифшица.

DOI: 10.21883/JTF.2019.09.48058.440-18

\section{Введение}

Дисперсионные силы [1], обусловленные вакуумными и тепловыми флуктуациями электромагнитного поля, вызывают в настоящее время большой интерес как в общетеоретическом плане, так и в связи с актуальными практическими приложениями. В простейшем случае двух параллельных пластин, разделенных вакуумной щелью шириной от нанометра до нескольких микрометров, это хорошо известные силы Ван-дер-Ваальса и Казимиpa [1-4]. В историческом плане эффект слипания близко расположенных материальных поверхностей приписывался именно действию сил Ван-дер-Ваальса, а эффект Казимира, первоначально обнаруженный для идеально проводящих пластин [5], рассматривался в основном с абстрактной, теоретико-полевой точки зрения [6,7]. Постепенно, однако, было осознано, что обе эти силы представляют собой две стороны одного и того же квантового явления, причем на расстояниях до нескольких нанометров результат зависит от постоянной Планка $\hbar$ (силы Ван-дер-Ваальса), а на бо́льших расстояниях, где начинают сказываться эффекты запаздывания электромагнитного взаимодействия, и от скорости света $c$ (силы Казимира).

Общая теория дисперсионных сил для случая двух материальных полупространств, разделенных щелью, была разработана Е.М. Лифшицем [8,9]. В настоящее время она обобщена на случай тел произвольной формы [4]. В рамках этой теории свободная энергия дисперсион- ного взаимодействия выражается в виде функционала от частотно-зависящих диэлектрической и магнитной проницаемостей взаимодействующих тел. Поставлены многочисленные прецизионные эксперименты по измерению дисперсионных сил, действующих как через вакуумную (см. обзоры $[10,11]$ и более поздние работы [12-17]), так и заполненную жидкостью щель [18-20]. Результаты проведенных экспериментальных и теоретических исследований использованы в атомно-силовой микроскопии, при изучении радиационной передачи тепла и атомного трения [21-24], а также при создании микроэлектромеханических устройств, действующих под совместным влиянием дисперсионных и электрических сил (см. обзор в [4]). В частности, было достигнуто понимание того, что при разработке новых поколений микроустройств с размером элементов менее микрометра будет необходимо не только учитывать наличие сил Ван-дер-Ваальса и Казимира, но и использовать данные силы в качестве основных факторов, определяющих функционирование устройства.

Независимо от вышесказанного большое внимание в последнее время привлекают различные электронные устройства, оптические модуляторы, переключатели и биосенсоры, использующие магнитную жидкость, заключенную между двумя материальными пластинами (см., например, обзор [25] и работы [26-29]). Магнитная жидкость является устойчивой коллоидной системой, состоящей из обычной жидкости (например, керосина или воды) и магнитных наночастиц, которые могут быть 
покрыты каким-либо поверхностно-активным веществом для предотвращения их слипания [30]. В рамках общей тенденции к миниатюризации уже созданы микроскопические устройства, где магнитная жидкость заключена между двумя близко расположенными поверхностями [31-34]. Поэтому возникает вопрос о роли дисперсионных сил в таких устройствах. Следует отметить, однако, что хотя силы Ван-дер-Ваальса и Казимира, действующие между двумя пластинами, разделенными обычной жидкостью, исследованы достаточно полно [4,9,18-20], случай магнитной промежуточной жидкости до сих пор не рассматривался. Принимая во внимание, что учет магнитных свойств ограничивающих щель пластин уже привел к обнаружению ряда интересных свойств дисперсионных сил [14-17,35,36], исследование случая щели, заполненной магнитной жидкостью, представляется весьма актуальным.

В настоящей работе представлены формализм теории Лифшица, позволяющий вычислять свободную энергию и давление дисперсионных сил в конфигурации двух параллельных пластин, разделенных щелью, которая заполнена магнитной жидкостью. Этот формализм применен к вычислению дисперсионного давления, возникающего между металлической $(\mathrm{Au})$ и диэлектрической $\left(\mathrm{SiO}_{2}\right)$ пластинами, разделенными коллоидной системой керосина и наночастиц магнетита $\left(\mathrm{Fe}_{3} \mathrm{O}_{4}\right)$. Исследованы зависимости давления от ширины щели, от процентного содержания магнитных наночастиц в жидкости и от диаметра этих наночастиц. Для этого установлено поведение диэлектрических свойств всех упомянутых выше материалов вдоль мнимой оси частот. Показано, что присутствие наночастиц приводит к значительному уменьшению величины дисперсионного давления, которая быстро падает также с ростом ширины щели. При фиксированных ширине щели и диаметре наночастиц отношение дисперсионных давлений в случаях магнитной жидкости и чистого керосина убывает с ростом концентрации наночастиц в магнитной жидкости. Наконец, показано, что при фиксированных ширине щели и процентном содержании магнитных наночастиц дисперсионное давление является медленно убывающей функцией их диаметра.

\section{1. Формализм теории Лифшица для трехслойной системы с магнитной промежуточной средой}

Рассмотрим две параллельные металлическую и диэлектрическую пластины, щель между которыми шириной $a$ заполнена магнитной жидкостью. Диэлектрические проницаемости металла, магнитной жидкости и диэлектрика обозначим $\varepsilon^{(1)}(\omega), \varepsilon(\omega)$ и $\varepsilon^{(2)}(\omega)$ соответственно, а магнитную проницаемость жидкости $\mu(\omega)$. Толщина пластин предполагается достаточно большой, чтобы их можно было рассматривать как полупространства. Это означает, что металлическая пласти- на (ниже будет рассматриваться Аu) должна быть толще, чем $100 \mathrm{~nm}$ [4], а диэлектрическая — толще, чем $2 \mu \mathrm{m}$ [37]. Вся система предполагается находящейся при температуре $T$ в состоянии теплового равновесия с окружающей средой. В этом случае, следуя теории Лифшица $[4,8,9]$, приходим к следующей формуле для свободной энергии флуктуационного поля на единицу площади пластин:

$$
\begin{aligned}
& \mathscr{F}(a, T)=\frac{k_{B} T}{2 \pi} \sum_{l=0}^{\infty}, \int_{0}^{\infty} k_{\perp} d k_{\perp} \\
& \quad \times \sum_{\alpha} \ln \left[1-r_{\alpha}^{(1)}\left(i \xi_{l}, k_{\perp}\right) r_{\alpha}^{(2)}\left(i \xi_{l}, k_{\perp}\right) e^{-2 a k\left(i \xi_{l}, k_{\perp}\right)}\right] .
\end{aligned}
$$

Здесь $k_{B}$ - это постоянная Больцмана, штрих у знака суммы по $l=0,1,2, \ldots$ означает, что член с $l=0$ делится на 2, частоты Мацубары имеют вид $\xi_{l}=2 \pi k_{B} T l / \hbar$, интегрирование проводится по абсолютной величине проекции волнового вектора на плоскость пластин, а

$$
k\left(i \xi_{l}, k_{\perp}\right)=\left[k_{\perp}^{2}+\varepsilon\left(i \xi_{l}\right) \mu\left(i \xi_{l}\right) \frac{\xi_{l}^{2}}{c^{2}}\right]^{1 / 2} .
$$

Оставшиеся неопределенными величины $r_{\alpha}^{(1,2)}$ имеют смысл коэффициентов отражения на первой и второй пластинах для двух независимых поляризаций электромагнитного поля $\alpha=\mathrm{TM}$ ( $s$-поляризация) в случае, когда вектор электрического поля перпендикулярен плоскости падения, и $\alpha=\mathrm{TE}$ ( $p$-поляризация), когда этот вектор параллелен плоскости падения. Коэффициенты отражения даются известными формулами Френеля, но в отличие от стандартных ситуаций вычисленными на чисто мнимых частотах Мацубары

$$
\begin{gathered}
r_{\mathrm{TM}}^{(n)}\left(i \xi_{l}, k_{\perp}\right)=\frac{\varepsilon^{(n)}\left(i \xi_{l}\right) k\left(i \xi_{l}, k_{\perp}\right)-\varepsilon\left(i \xi_{l}\right) k^{(n)}\left(i \xi_{l}, k_{\perp}\right)}{\varepsilon^{(n)}\left(i \xi_{l}\right) k\left(i \xi_{l}, k_{\perp}\right)+\varepsilon\left(i \xi_{l}\right) k^{(n)}\left(i \xi_{l}, k_{\perp}\right)}, \\
r_{\mathrm{TE}}^{(n)}\left(i \xi_{l}, k_{\perp}\right)=\frac{k\left(i \xi_{l}, k_{\perp}\right)-\mu\left(i \xi_{l}\right) k^{(n)}\left(i \xi_{l}, k_{\perp}\right)}{k\left(i \xi_{l}, k_{\perp}\right)+\mu\left(i \xi_{l}\right) k^{(n)}\left(i \xi_{l}, k_{\perp}\right)},
\end{gathered}
$$

где $n=1,2$ и

$$
k^{(n)}\left(i \xi_{l}, k_{\perp}\right)=\left[k_{\perp}^{2}+\varepsilon^{(n)}\left(i \xi_{l}\right) \frac{\xi_{l}^{2}}{c^{2}}\right]^{1 / 2} .
$$

Дисперсионная сила, действующая на единицу площади пластин, т. е. давление, определяется как

$$
P(a, T)=-\frac{\partial \mathscr{F}(a, T)}{\partial a} .
$$

Подставляя (1) в (5) и дифференцируя по $a$, получаем искомое выражение для силы притяжения на единицу площади пластин, разделенных магнитной жидкостью

$$
\begin{aligned}
P(a, T)= & -\frac{k_{B} T}{\pi} \sum_{l=0}^{\infty}, \int_{0}^{\infty} k_{\perp} d k_{\perp} k\left(i \xi_{l}, k_{\perp}\right) \\
& \times \sum_{\alpha}\left[\frac{e^{2 a k\left(i \xi_{l}, k_{\perp}\right)}}{r_{\alpha}^{(1)}\left(i \xi_{l}, k_{\perp}\right) r_{\alpha}^{(2)}\left(i \xi_{l}, k_{\perp}\right)}-1\right]^{-1} .
\end{aligned}
$$


Расчет дисперсионного давления требует знания значений диэлектрических проницаемостей пластин и магнитной жидкости, а также магнитной проницаемости этой жидкости на чисто мнимых частотах Мацубары. Эти величины найдены в разд. 2.

\section{2. Диэлектрические и магнитные свойства материалов вдоль мнимой оси частот}

Диэлектрические проницаемости золота и кварцевого стекла вдоль мнимой оси частот хорошо изучены в литературе. Так, проницаемость кварцевого стекла, $\varepsilon^{(2)}(i \xi)$, как функция $\xi / \xi_{1}$, показана нижней сплошной линией на рис. 1, $a$ [38], начиная с первой частоты Мацубары. При этом $\varepsilon^{(2)}(0)=3.801$.

Диэлектрическая проницаемость золота вдоль мнимой оси частот $\varepsilon^{(2)}(i \xi)$ получена с использованием оптических данных для комплексного показателя преломления и соотношения Крамерса-Кронига (см., например, $[4,10])$. При этом, поскольку оптические данные [39] доступны только при $\hbar \omega \geq 0.125 \mathrm{eV}$, необходимо экс-
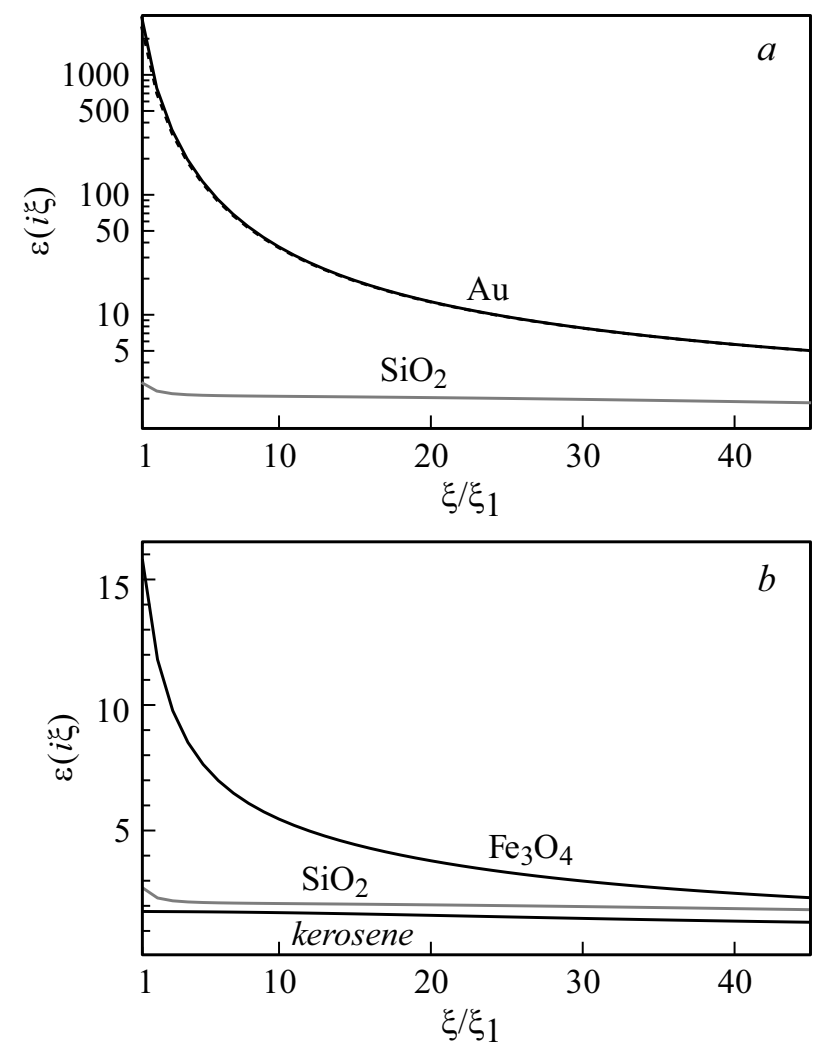

Pис. 1. Диэлектрическая проницаемость как функция отношения частоты вдоль мнимой оси к частоте Мацубары с номером $l=1$ показана $(a)$ верхними сплошной и штриховой линиями для золота при использовании экстраполяции данных посредством плазменной и Друде моделей соответственно, а также нижней сплошной линией для кварцевого стекла и $(b)$ верхней, средней и нижней линиями для магнетита, кварцевого стекла и керосина соответственно. траполировать их в область низких частот. Такая экстраполяция выполнена разными авторами с помощью плазменной и Друде моделей (см. обзор в $[4,10])$. При этом оказалось, что хотя использование Друде модели может показаться более обоснованным, так как учитывает процессы релаксации свободных электронов в реальных полях, получаемые с ее помощью результаты для дисперсионных сил исключаются экспериментом с абсолютной достоверностью [4,10,15-17]. В то же время было показано, что расчеты дисперсионных сил, использующие экстраполяцию с помощью плазменной модели, находятся в полном согласии с экспериментальными данными.

В области чисто мнимых частот $\xi \geq \xi_{1}$ диэлектрическая проницаемость $\varepsilon^{(2)}(i \xi)$ показана верхней сплошной линией и штрихованной линией на рис. 1, a при использовании плазменной и Друде экстраполяций соответственно. Как видно из рис. $1, a$, при $\xi \geq \xi_{1}$ данные линии почти совпадают. При стремлении $\xi$ к нулю, однако, различие между ними быстро увеличивается поскольку $\varepsilon^{(1)}(i \xi) \approx \omega_{p}^{2} / \xi^{2}$ в случае экстраполяции оптических данных плазменной моделью и $\approx \omega_{p}^{2} /(\xi \gamma)$, если используется экстраполяция моделью Друде, где $\omega_{p}$ плазменная частота, а $\gamma$ - параметр релаксации (для золота $\left.\omega_{p}=1.37 \cdot 10^{16} \mathrm{rad} / \mathrm{s}, \gamma=5.3 \cdot 10^{14} \mathrm{rad} / \mathrm{s}[39]\right)$. В peзультате на нулевой частоте при использовании обеих моделей из (3) получается один и тот же результат $r_{\mathrm{TM}}^{(1)}\left(0, k_{\perp}\right)=1$. Для ТЕ-поляризации, однако, в случае экстраполяции плазменной моделью из (3) следует

$$
r_{\mathrm{TE}}^{(1)}\left(0, k_{\perp}\right)=\frac{c k_{\perp}-\mu(0) \sqrt{c^{2} k_{\perp}^{2}+\omega_{p}^{2}}}{c k_{\perp}+\mu(0) \sqrt{c^{2} k_{\perp}^{2}+\omega_{p}^{2}}},
$$

в то время как экстраполяция Друде моделью ведет к

$$
r_{\mathrm{TE}}^{(1)}\left(0, k_{\perp}\right)=\frac{1-\mu(0)}{1+\mu(0)} .
$$

Для диэлектрической пластины на нулевой частоте имеем

$$
\begin{gathered}
r_{\mathrm{TM}}^{(2)}\left(0, k_{\perp}\right)=\frac{\varepsilon^{(2)}(0)-\varepsilon(0)}{\varepsilon^{(2)}(0)+\varepsilon(0)}, \\
r_{\mathrm{TE}}^{(2)}\left(0, k_{\perp}\right)=\frac{1-\mu(0)}{1+\mu(0)} .
\end{gathered}
$$

Для магнетита в литературе отсутствуют данные о значении его диэлектрической проницаемости вдоль мнимой оси частот. Можно, однако, воспользоваться измеренными значениями мнимой части диэлектрической проницаемости магнетита $\operatorname{Im} \varepsilon^{(m)}(\omega)$ в интервале от $\omega_{1}=2 \cdot 10^{14} \mathrm{rad} / \mathrm{s}$ до $\omega_{2}=1.8 \cdot 10^{16} \mathrm{rad} / \mathrm{s}[40]$ и экстраполировать их в область $\omega<\omega_{1}$ с помощью модели Дебая

$$
\operatorname{Im} \varepsilon^{(m)}(\omega)=\frac{C_{D} \omega_{D} \omega}{\omega_{D}^{2}+\omega^{2}} .
$$

Из условия гладкого сшивания измерительных данных с экстраполяцией (10) легко находим $C_{D}=24.02$ и 
$\omega_{D}=2.05 \cdot 10^{14} \mathrm{rad} / \mathrm{s}$. В области высоких частот экстраполяция осуществляется обычным образом, т. е. как

$$
\operatorname{Im} \varepsilon^{(m)}(\omega)=C\left(\frac{\omega_{2}}{\omega}\right)^{3}
$$

где значение $C=1.58$ получается из условия сшивания с измеренными значениями мнимой части проницаемости при высоких частотах.

Подставляя (10) и (11) в правую часть соотношения Крамерса-Кронига [4] и производя интегрирование в областях низких и высоких частот, получаем

$$
\begin{gathered}
\varepsilon^{(m)}(i \xi)=1+\frac{2 C_{D} \omega_{D}^{2}}{\pi\left(\xi^{2}-\omega_{D}^{2}\right)}\left[\frac{\xi}{\omega_{D}} \arctan \frac{\omega_{1}}{\xi}-\arctan \frac{\omega_{1}}{\omega_{D}}\right] \\
+\frac{2}{\pi} \int_{\omega_{1}}^{\omega_{2}} \frac{\omega \operatorname{Im} \varepsilon^{(m)}(\omega)}{\xi^{2}+\omega^{2}}+\frac{2 C \omega_{2}^{2}}{\pi \xi^{2}}\left(1+\frac{\omega_{2}}{\xi} \arctan \frac{\omega_{2}}{\xi}-\frac{\pi}{2}\right) .
\end{gathered}
$$

Диэлектрическая проницаемость магнетита (12), как функция $\xi / \xi_{1}$, показана верхней линией на рис. $1, b$.

Хотя керосин часто используется как основа магнитных жидкостей $[41,42]$, его диэлектрические свойства исследованы недостаточно полно. Можно, однако, воспользоваться результатами измерений мнимой части диэлектрической проницаемости керосина в микроволновой [41] и инфракрасной областях [43]. С помощью соотношения Крамерса-Кронига представим проницаемость керосина вдоль мнимой оси в виде

$$
\varepsilon^{(k)}(i \xi)=1+\frac{B}{1+\xi \tau}+\frac{C_{\mathrm{IR}}}{1+\left(\xi / \omega_{\mathrm{IR}}\right)^{2}}+\frac{C_{\mathrm{UV}}}{1+\left(\xi / \omega_{\mathrm{UV}}\right)^{2}},
$$

где второй, третий и четвертый члены в правой части (13) учитывают вклады ориентации постоянных диполей, а также ионной и электронной поляризации соответственно. Используя данные [41], находим $B=0.020$ и $1 / \tau=8.0 \cdot 10^{8} \mathrm{rad} / \mathrm{s}$, а из данных [43] получаем $C_{\mathrm{IR}}=0.007$ и $\omega_{\mathrm{IR}}=2.14 \cdot 10^{14} \mathrm{rad} / \mathrm{s}$. Значения параметров в ультрафиолетовой области $C_{\mathrm{UV}}=0.773$ и $\omega_{\mathrm{UV}}=1.0 \cdot 10^{16} \mathrm{rad} / \mathrm{s}$ находим, следуя [38], с использованием известного значения $\varepsilon^{(k)}(0)=1.8$ [41]. Полученная проницаемость (13) как функция $\xi / \xi_{1}$ показана нижней линией ни рис. $1, b$, где для удобства сравнения в однородном масштабе повторена также и проницаемость кварцевого стекла.

Ниже предполагается, что наночастицы магнетита представляют собой сферы диаметра $d \ll \lambda$, где $\lambda-$ это характерная для данной задачи длина волны. В диапазоне расстояний $a \geq 100 \mathrm{~nm}$ дающие основной вклад в (6) частоты $\xi \leq 10^{16} \mathrm{rad} / \mathrm{s}$, т.е. $\lambda \geq 180 \mathrm{~nm}$. Поэтому рассматриваемые ниже наночастицы с $d<10 \mathrm{~nm}$ вполне удовлетворяют сделанному предположению. Можно допустить также, что диэлектрическая функция поверхностно-активного вещества близка по своим свойствам к проницаемости исходной жидкости. При этих условиях диэлектрическая проницаемость магнитной жидкости $\varepsilon(i \xi)$ может быть получена из формулы Рэлея [44]

$$
\frac{\varepsilon(i \xi)-\varepsilon^{(k)}(i \xi)}{\varepsilon(i \xi)+2 \varepsilon^{(k)}(i \xi)}=\Phi \frac{\varepsilon^{(m)}(i \xi)-\varepsilon^{(k)}(i \xi)}{\varepsilon^{(m)}(i \xi)+2 \varepsilon^{(k)}(i \xi)},
$$

где $\Phi$ - объемная доля наночастиц магнетита в магнитной жидкости.

В заключение разд. 2 остается рассмотреть магнитную проницаемость магнитной жидкости $\mu(i \xi)$. Поскольку магнитные свойства влияют на дисперсионные силы только через члены (1) и (6) с $l=0$ [35], достаточно рассмотреть только

$$
\mu(0)=1+4 \pi \chi(0),
$$

где начальная восприимчивость парамагнитной (суперпарамагнитной) системы представляется как [45]

$$
\chi(0)=N \frac{M^{2}}{3 k_{B} T}
$$

Здесь $N=\Phi / V$, а магнитный момент $M=M_{s} V$ однодоменной наночастицы объема $V=\pi d^{3} / 6$ выражается через намагниченность насыщения на единицу объема $M_{s} \approx 300 \mathrm{emu} / \mathrm{cm}^{3}$ [46]. Тогда из (15) и (16) получаем

$$
\mu(0)=1+\frac{2 \pi^{2} \Phi}{9} \frac{M_{s}^{2} d^{3}}{k_{B} T} .
$$

В качестве примера при $T=300 \mathrm{~K}$ и $\Phi=0.05$ получаем $\mu(0) \approx 1.03$ и 1.24 в случаях наночастиц магнетита с диаметром 5 и $10 \mathrm{~nm}$ соответственно.

\section{3. Давление дисперсионной силы в зависимости от расстояния между пластинами, объемной доли и диаметра наночастиц}

С использованием (3) и (6), а также результатов разд. 2 для диэлектрической (14) и магнитной (17) проницаемостей магнитной жидкости было рассчитано давление дисперсионной силы между пластинами из золота и кварцевого стекла, ограничивающими эту жидкость, в зависимости от расстояния между ними. При этом использовались численные данные обеих моделей диэлектрической проницаемости золота и диэлектрической проницаемости стекла (см. рис. 1). Результаты расчета при $\Phi=0.05, d=10 \mathrm{~nm}$ показаны верхней сплошной и штриховой линиями на рис. 2 в случаях, когда экстраполяция оптических данных золота в область низких частот проводилась с помощью плазменной и Друде моделей соответственно. Как видно из рис. 2, в данном случае результаты, полученные с помощью обеих экстраполяций, различаются незначительно. На том же рисунке нижней сплошной линией показано дисперсионное давление между теми же пластинами, разделенными чистым керосином (без примеси наночастиц 


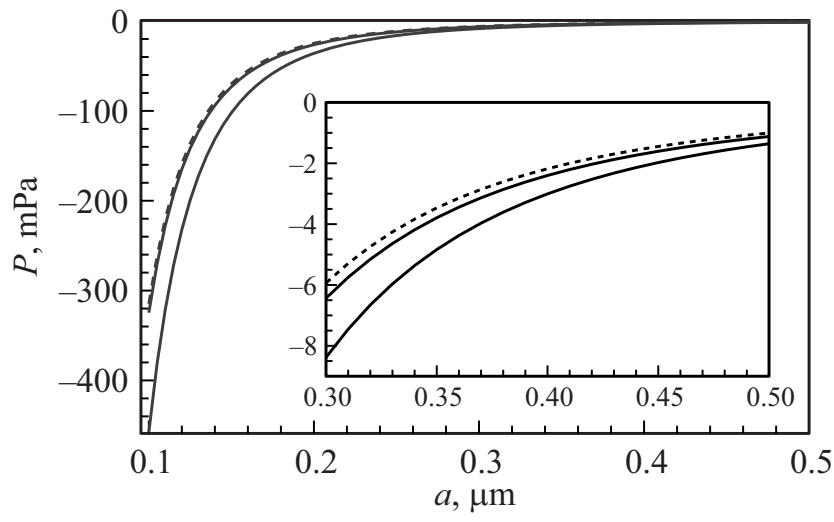

Pис. 2. Дисперсионное давление между пластинами из золота и кварцевого стекла, разделенными магнитной жидкостью на основе керосина с 5-процентным содержанием наночастиц магнетита диаметром $10 \mathrm{~nm}$, показано как функция расстояния между стенками верхней сплошной и штриховой линиями при использовании экстраполяции данных посредством плазменной и Друде моделей соответственно. Случай чистого керосина между теми же стенками представлен нижней сплошной линией. На вставке в увеличенном масштабе показана область бо́льших расстояний.

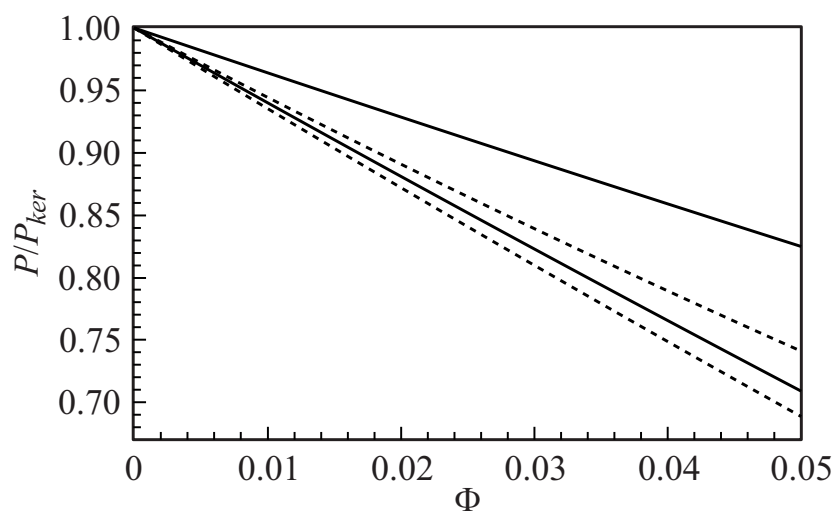

Pис. 3. Отношение дисперсионных давлений между пластинами из золота и кварцевого стекла, разделенными магнитной жидкостью на основе керосина и наночастиц магнетита диаметром $10 \mathrm{~nm}$ и чистым керосином, показано как функция доли наночастиц сплошными и штриховыми линиями при использовании экстраполяции данных посредством плазменной и Друде моделей соответственно. Нижняя и верхняя пары сплошных и штриховых линий построены для расстояния между пластинами $a=100$ и $500 \mathrm{~nm}$ соответственно.

магнетита). Согласно полученному результату, присутствие магнитных наночастиц значительно (в 1.44 раза для $a=0.1 \mu \mathrm{m})$ уменьшает величину дисперсионного давления. Это объясняется влиянием диэлектрической проницаемости магнитной жидкости, в результате чего существенно уменьшается ТМ-коэффициент отражения на диэлектрической стенке.

С использованием тех же выражений из разд. 1 и 2 была исследована зависимость дисперсионного давления между золотой и стеклянной пластинами, разделенными магнитной жидкостью, в случае наночастиц диаметром $d=10 \mathrm{~nm}$ и фиксированного расстояния между пластинами от объемной доли наночастиц Ф. Результаты вычисления, нормированные на давление между теми же пластинами, разделенными чистым керосином, представлены на рис. 3 двумя парами сплошных и штриховых линий, полученных при расстоянии между пластинами $a=100$ и $500 \mathrm{~nm}$ для нижней и верхней пары соответственно. Как и выше, сплошные и штриховые линии были рассчитаны с помощью экстраполяции оптических данных золота в область низких частот плазменной и Друде моделью соответственно. Как видно из рис. 3, с увеличением доли наночастиц в магнитной жидкости происходит монотонное уменьшение величины дисперсионного давления по сравнению со случаем чистого керосина. Это уменьшение более значительно на меньших расстояниях между пластинами. Кроме того, рис. 3 показывает, что на бо́льших расстояниях между пластинами использование различных экстраполяций оптических данных золота приводит к существенно различающимся результатам. Физически указанное поведение линий на рис. 3 объясняется ростом магнитной проницаемости (17) на нулевой частоте с ростом $\Phi$.

Наконец, полученные в разд. 1 и 2 результаты были использованы для расчета зависимости дисперсионного давления от диаметра наночастиц магнетита. Данные расчеты были проведены при расстоянии между пластинами $a=200 \mathrm{~nm}$. Полученные результаты представлены на рис. 4 тремя парами сплошных и штриховых линий, построенных снизу вверх для случаев объемной концентрации наночастиц $\Phi=0.01,0.03$ и 0.05 соответственно. Сплошная и штриховая линии означают использование указанных выше экстраполяций оптических данных золота. Как видно из рис. 4, дисперсионное давление

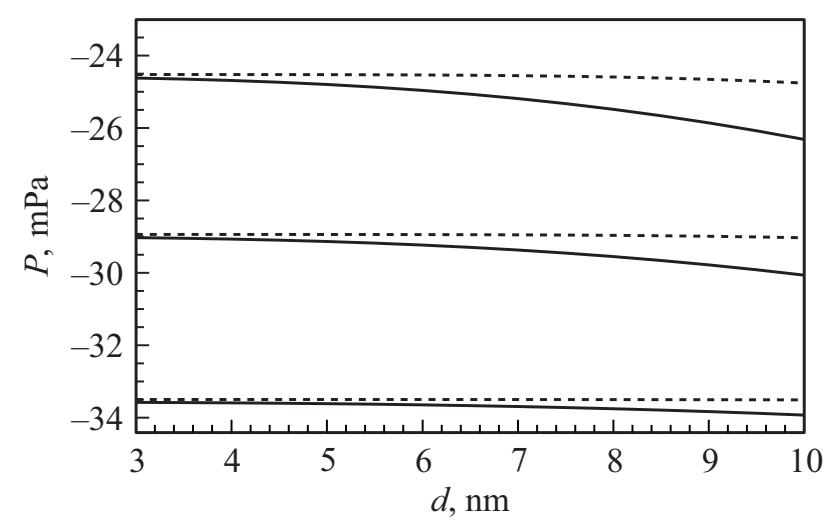

Рис. 4. Дисперсионное давление между пластинами из золота и кварцевого стекла на расстоянии $200 \mathrm{~nm}$, разделенными магнитной жидкостью на основе керосина и наночастиц магнетита, показано как функция их диаметра сплошными и штриховыми линиями при использовании экстраполяции данных посредством плазменной и Друде моделей соответственно. Нижняя, средняя и верхняя пары сплошных и штриховых линий построены для доли наночастиц в магнитной жидкости $\Phi=0.01,0.03$ и 0.05 соответственно. 
убывает с ростом $d$. Данное убывание ускоряется с ростом $\Phi$, который приводит также к увеличению зависимости от типа экстраполяции оптических данных золота. Физически особенности линий на рис. 4 объясняются ростом магнитной проницаемости на нулевой частоте с увеличением $d$.

До сих пор рассматривались расстояния между пластинами менее микрометра. Отметим, что на расстояниях, превышающих примерно $2.5 \mu \mathrm{m}$, основной вклад в (6) дает член с $l=0$. В этом случае, т.е. в так называемом классическом пределе, можно получить простые аналитические выражения для дисперсионного давления в системе с магнитной жидкостью. Эти выражения различны в зависимости от используемой экстраполяции оптических данных золота. Так, подставляя в член (6) с $l=0$ выражения (8) и (9) и интегрируя, в случае экстаполяции посредством Друде модели получаем

$$
\begin{aligned}
& P_{D}(a, T)=-\frac{k_{B} T}{8 \pi a^{3}} \\
& \times\left\{\operatorname{Li}_{3}\left[\frac{\varepsilon^{(2)}(0)-\varepsilon(0)}{\varepsilon^{(2)}(0)+\varepsilon(0)}\right]+\operatorname{Li}_{3}\left[\left(\frac{1-\mu(0)}{1+\mu(0)}\right)^{2}\right]\right\},
\end{aligned}
$$

где $\mathrm{Li}_{3}(z)$ - полилогарифм порядка 3.

При использовании экстраполяции данных посредством плазменной модели, подставляем в член (6) с $l=0$ выражения (7) и (9). В этом случае точное вычисление интеграла оказывается невозможным. Можно, однако, воспользоваться разложением по параметру

$$
\frac{\delta}{\mu(0) a} \ll 1,
$$

где $\delta=c / \omega_{p} \approx 21.9 \mathrm{~nm}$ имеет смысл глубины проникновения электромагнитного поля в золото. В результате получаем

$$
\begin{aligned}
& P_{p}(a, T)=-\frac{k_{B} T}{8 \pi a^{3}}\left\{\operatorname{Li}_{3}\left[\frac{\varepsilon^{(2)}(0)-\varepsilon(0)}{\varepsilon^{(2)}(0)+\varepsilon(0)}\right]\right. \\
& \left.+\mathrm{Li}_{3}\left[\left|\frac{1-\mu(0)}{1+\mu(0)}\right|\right]\left[1-3 \frac{\delta}{\mu(0) a}+6\left(\frac{\delta}{\mu(0) a}\right)^{2}\right]\right\} .
\end{aligned}
$$

При расстояниях между пластинами, превышающих $2.5 \mu \mathrm{m},(18)$ и (20) дают значения дисперсионного давления, совпадающие с результатами численных расчетов по (6) с точностью до доли процента.

\section{Заключение}

Выше исследовано дисперсионное давление между золотой и стеклянной пластинами, разделенными тонким слоем магнитной жидкости, которая представляет собой коллоидную систему керосина и наночастиц магнетита сферической формы. Как отмечено во Введении, трехслойные системы типа металл-магнитная жидкостьдиэлектрик находят широкое применение в различных микроустройствах. Учитывая тот факт, что дисперсионные силы быстро растут с уменьшением размера устройства и могут оказывать как полезное, так и вредное воздействие на его функционирование, исследование физических процессов, определяемых электромагнитными флуктуациями, является актуальным при разработке новых поколений такого рода устройств.

В работе формализм теории Лифшица представлен для случая, когда в качестве промежуточного слоя используется магнитная жидкость. Численные расчеты дисперсионного давления проведены для золотой и стеклянной пластин, заключающих магнитную жидкость на основе керосина и наночастиц магнетита, при комнатной температуре. Для этого были использованы известные диэлектрические проницаемости золота и кварцевого стекла вдоль мнимой оси частот, а также с использованием имеющихся в литературе оптических данных построены соответствующие представления для диэлектрических функций магнетита, керосина и для магнитной проницаемости магнетита.

В результате было детально исследовано поведение дисперсионного давления в зависимости от расстояния между пластинами, от объемной доли наночастиц в магнитной жидкости и от их диаметра. Полученные закономерности нашли простое физическое объяснение. В области расстояний между пластинами более $2.5 \mu \mathrm{m}$ выведены удобные аналитические формулы. Предложенный формализм может в дальнейшем применяться в конфигурациях с различными материалами пластин, компонентами магнитной жидкости, а также при произвольной температуре.

\section{Конфликт интересов}

Авторы заявляют, что у них нет конфликта интересов.

\section{Список литературы}

[1] Mahanty J., Ninham B.W. Dispersion Forces. London: Academic Press, 1976. 236 p.

[2] Бараш Ю.С. Силы Ван-дер-Ваальса. М.: Наука, 1988. $344 \mathrm{c}$.

[3] Parsegian V.A. Van der Waals Forces: A Handbook for Biologists, Chemists, Engineers, and Physicists. Cambridge: Cambridge University Press, 2005. 380 p.

[4] Bordag M., Klimchitskaya G.L., Mohideen U., Mostepanenko V.M. Advances in the Casimir Effect. Oxford: Oxford University Press, 2015. 776 p.

[5] Casimir H.B.G. // Proc. Kon. Ned. Akad. Wet. B. 1948. Vol. 51. N 7. P. 793-795.

[6] Мостепаненко В.М., Трунов Н.Н. // УФН. 1988. Т. 156. Вып. 3. C. 385-426. [Mostepanenko V.M., Trunov N.N. // Sov. Physics-Uspekhi. 1988. Vol. 31. N 11. P. 965-987.]

[7] 7 Мостепаненко В.М., Трунов Н.Н. Эффект Казимира и его приложения. М.: Энергоатомиздат, 1990. 215 с. [Mostepanenko V.M., Trunov N.N. The Casimir Effect and its Applications. Oxford: Oxford University Press, 1998. 199 p.] 
[8] Лифиии, Е.М. // ЖЭТФ. 1955. Т. 29. Вып. 1. С. 94-110. [Lifshitz E.M. // Sov. Phys. JETP. 1956. Vol. 2. N 1. P. 73-83.]

[9] Дзялошинский И.Е., Лифиии, Е.М., Питаевский Л.П. // УФН. 1961. Т. 73. Вып. 3. С. 381-422. [Dzyaloshinskii I.E., Lifshitz E.M., Pitaevskii L.P. // Adv. Phys. 1961. Vol. 10. N 3. P. 165-209.]

[10] Klimchitskaya G.L., Mohideen U., Mostepanenko V.M. // Rev. Mod. Phys. 2009. Vol. 81. N 4. P. 1827-1885.

[11] Klimchitskaya G.L., Mohideen U., Mostepanenko V.M. // Int. J. Mod. Phys. B. 2011. Vol. 25. N 2. P. 171-230.

[12] Chang C.-C., Banishev A.A., Klimchitskaya G.L., Mostepanenko V.M., Mohideen U. // Phys. Rev. Lett. 2011. Vol. 107. N 090403. P. 1-4.

[13] Banishev A.A., Chang C.-C., Castillo-Garza R., Klimchitskaya G.L., Mostepanenko V.M., Mohideen U. // Phys. Rev. B. 2012. Vol. 85. N 045436. P. 1-18.

[14] Banishev A.A., Chang C.-C., Klimchitskaya G.L., Mostepanenko V.M., Mohideen U. // Phys. Rev. B. 2012. Vol. 85. N 195422. P. 1-7.

[15] Banishev A.A., Klimchitskaya G.L., Mostepanenko V.M., Mohideen U. // Phys. Rev. Lett. 2013. Vol. 110. N 137401. P. 1-5.

[16] Banishev A.A., Klimchitskaya G.L., Mostepanenko V.M., Mohideen U. // Phys. Rev. B. 2013. Vol. 88. N 155410. P. $1-20$.

[17] Bimonte G., López D., Decca R.S. // Phys. Rev. B. 2016. Vol. 93. N 184434. P. 1-15.

[18] Munday J.N., Capasso F., Parsegian V.A., Bezrukov S.M. // Phys. Rev. A. 2008. Vol. 78. N 032109. P. 1-8.

[19] Munday J.N., Capasso F., Parsegian V.A. // Nature. 2009. Vol. 457. N 7226. P. 170-173.

[20] Rodriguez A.W., Capasso F., Johnson S.G. // Nat. Photon. 2011. Vol. 5. N 4. P. 211-221.

[21] Благов Е.В., Моисеев Ю.Н., Мостепаненко В.М., Мусатенко А.Ю., Панов В.И., Савинов С.В., Соколов И.Ю. // ЖТФ. 1994. Т. 64. Вып. 1. С. 89-102. [Blagov E.V., Moiseev Yu.N., Mostepanenko V.M., Musatenko A.Yu., Panov V.I., Savinov S.V., Sokolov I.Yu. // Tech. Phys. 1994. Vol. 39. N 1. P. 49-56.]

[22] Моисеев Ю.Н., Мостепаненко В.М., Панов В.И., Соколов И.Ю. // ЖТФ. 1990. Т. 60. Вып. 1. С. 141148. [Moiseev Yu.N., Mostepanenko V.M., Panov V.I., Sokolov I.Yu. // Tech. Phys. 1990. Vol. 35. N 1. P. 84-88.]

[23] Волокитин А.И., Перссон Б.Н.Дж. // УФН. 2007. Т. 177. Вып. 9. C. 921-951. [Volokitin A.I., Persson B.N.J. // PhysicsUspekhi. 2007. Vol. 50. N 9. P. 879-906.]

[24] Дедков Г.В., Кясов А.А. // ЖТФ. 2014. Т. 84. Вып. 4. С. 148 151. [Dedkov G.V., Kyasov A.A. // Tech. Phys. 2014. Vol. 59. N 4. P. 616-620.]

[25] Philip J., Laskar J.M. // J. Nanofluids. 2012. Vol. 1. N 1. P. 320.

[26] Mao L., Elborai S., He X., Zahn M., Koser H. // Phys. Rev. B. 2011. Vol. 84. N 104431. P. 1-7.

[27] Lin W., Miao Y., Zhang H., Liu B., Liu Y., Song B. // Appl. Phys. Lett. 2013. Vol. 103. N 151101. P. 1-4.

[28] Prokofiev A.V., Nepomnyashchaya E.K., Pleshakov I.V., Kuzmin Yu.I., Velichko E.N., Aksenov E.T. In: Next Generation Networks and Systems / Ed. O. Galinina, S. Balandin, Y. Koucheryavy. Springer, Cham. 2016. P. 680.

[29] Nepomnyashchaya E.K., Velichko E.N., Pleshakov I.V., Aksenov E.T., Savchenko E.A. // J. Phys.: Conf. Ser. 2017. Vol. 841. N 012020. P. 1-6.
[30] Rosensweig R.E. Ferrohydrodynamics. Cambridge: Cambridge University Press, 1985. 344 p.

[31] Goubault C., Jop P., Fermigier M., Baudry J., Bertrand E., Bibette J. // Phys. Rev. Lett. 2003. Vol. 91. N 260802. P. 1-4.

[32] Pekas N., Porter M.D., Tondra M., Popple A., Jander A. // Appl. Phys. Lett. 2004. Vol. 85. N 20. P. 4783-4785.

[33] Inglis D.W., Riehn R., Austin R.H., Sturm J.C. // Appl. Phys. Lett. 2004. Vol. 85. N 21. P. 5093-5095.

[34] Saga V., Nakamura T. // J. Appl. Phys. 2002. Vol. 91. N 10. P. 7003-7005.

[35] Geyer B., Klimchitskaya G.L., Mostepanenko V.M. // Phys. Rev. B. 2010. Vol. 81. N 104101. P. 1-15.

[36] Klimchitskaya G.L., Mostepanenko V.M. // Phys. Rev. B. 2016. Vol. 94. N 045404. P. 1-13.

[37] Klimchitskaya G.L., Mostepanenko V.M. // Phys. Rev. A. 2014. Vol. 89. N 052512. P. 1-7.

[38] Hough D.B., White L.R. // Adv. Colloid. Interface Sci. 1980. Vol. 14. N 1. P. 3-41.

[39] Handbook of Optical Constants of Solids / Ed. by E.D. Palik. NY.: Academic Press, 1980. 785 p.

[40] Schlegel A., Alvarado S.F., Wachter P. // J. Phys. C: Solid State Phys. 1979. Vol. 12. N 6. P. 1157-1164.

[41] Fannin P.C., Marin C.N., Malaescu I., Stefu N. // J. Phys.: Condens. Matter. 2007. Vol. 19. N 036104. P. 1-8.

[42] Hong C.-Y., Jang I.J., Horng H.E., Hsu C.J., Yao Y.D., Yang H.C. // J. Appl. Phys. 1997. Vol. 81. N 8. P. 4275-4277.

[43] Qi H., Zhang X., Jiang M., Wang Q., Li D. // Optik. 2016. Vol. 127. N 20. P. 8899-8906.

[44] Sihvola A.H. Electromagnetic Mixing Formulas and Applications. London: The Institution of Electrical Engineers, 1999. 284 p.

[45] Вонсовский С.В. Магнетизм. М.: Наука, 1971. 1032 с. [Vonsovskii S.V. Magnetism. N. Y.: Wiley, 1974. 776 p.]

[46] van Berkum S., Dee J.T., Philipse A.P., Erné B.E. // Int. J. Mol. Sci. 2013. Vol. 14. N 5. P. 10162-10177. 